\title{
Flexible Electronics Non-Destructive Uniformity Characterization by Synchronized Thermography
}

\author{
Kari Remes \\ Optoelectronics and Measurement \\ Techniques Research Unit \\ University of Oulu \\ Oulu, Finland \\ kari.remes@oulu.fi
}

\author{
Antti Latomäki \\ Optoelectronics and Measurement \\ Techniques Research Unit \\ University of Oulu \\ Oulu, Finland \\ antti.latomaki@oulu.fi
}

\author{
Tapio Fabritius \\ Optoelectronics and Measurement \\ Techniques Research Unit \\ University of Oulu \\ Oulu, Finland \\ tapio.fabritius@oulu.fi
}

\begin{abstract}
Synchronized thermography (ST) is utilized in the characterization of a flexible hybrid electronics. ST is used for both in the analysis of the structural uniformity and electrical functionality. Flexible lighting foils combining conventionally manufactured light-emitting diode (LED) chips and current regulators with a printed wiring on polyethylene terephthalate (PET) substrate were used as test samples. The measurement results proves that ST measurement method is a feasible method for the uniformity and electrical functionality characterization of large-area printed thin films with conventional electronics. ST is an interesting option to be used as a measurement tool for online quality monitoring of hybrid electronics manufacturing.
\end{abstract}

Keywords-infrared imaging, uniformity, optical measurement techniques, printed electronics, quality control

\section{INTRODUCTION}

Novel applications of thin, flexible, and large area platforms are emerging in signage and lighting solutions. Due to the development of materials and electronics manufacturing technologies, flexible hybrid electronics technology is nowadays providing an attractive possibility for implementing of flexible or even conformable lighting elements to be used in applications such as automotive and architecture. This technology provides more freedom to design and make very thin, large area lighting elements that can be shaped in different forms. Due to the current maturity level of technology, the conventionally fabricated LED chips bonded on printed flexible substrates are mainly used in these type of products but also organic light-emitting diodes OLEDs are expected to be exploited in such large area electronics based luminaires in the future [1].

In order to optimize and up-scale the manufacturing processes of hybrid electronics technology, including the lightning and signature devices, simple online compatible quality monitoring tools are needed. Optical characterization methods have shown to provide a non-destructive way to measure online, which is necessary when large area, high yield process monitoring is considered. Especially, the devices capable to test and analyze the functional properties of flexible hybrid electronics products are needed in industry commercializing the technology.

Previous research has shown that the combination of infrared (IR) imaging with electrical heating is an effective method for thin film uniformity characterization. In lock-in thermography technique, the samples are heated with a

The last author of this paper is financially supported by Academy of Finland's 6Genesis Flagship (grant no. 318927) and Academy of Finland's FIRI funding (grant no. 320017). modulated electrical signal and IR imaging is synchronized with heating to analyze temperature variation of the samples [2]. However, this technique is unnecessarily complex making it impractical to be utilized in real online production environments. Recently introduced simplified version of lock-in thermography, so called synchronized thermography (ST), is another IR imaging based characterization method [3]. In that case, electrical heating of the sample with direct current (DC) power is synchronized with IR imaging to produce thermograms, which are influenced by the nonuniformities of conductive thin films. Non-uniformities on electrical conductivity will influence on the current density, varying the temperature around it. Advantage of this approach is that only a very simple data processing is needed to localize and categorize defects on samples. ST has already shown to be a versatile technique to characterize the uniformity of thin films structures. It is shown to provide even quantitative information about the conductivity online in R2R environment [3-8].

In this study, the performance and applicability of the ST technique for patterned conductive structure with functional hybrid electronics (bonded regulators and LED chips) on it, is investigated and reported. In addition, the possibilities for the further development needs of ST technique are discussed.

\section{MATERIALS AND METHODS}

\section{A. Flexible Electronics Device as a test sample}

Two different flexible electronics samples were investigated in this study. The first sample (Sample 1) is a kind of intermediate product used as basis for the other sample, which is a functional LED lighting element (Sample $2)$. Both sample types are based on roll-to-roll (R2R) printed conductive silver ink (Asahi 411AW) and dielectric Henkel/Acheson (Electrodag 452SS and Electrodag PF455B) on 125- $\mu$ m-thick PET Melinex ST506 substrate. Luxeon 3535L LED chips were bonded on printed foil with adhesive glue (Sample 2). Continuous and mass customized wiring board layout is design to be compatible with R2R screen printing technique so that crossing sections of wiring conduits were enabled without short circuit by printing a dielectric pattern between the lower and upper conductor patterns in crossing sections shown in Fig 1. More detailed description of the sample design and manufacturing process is available in ref [1]. 


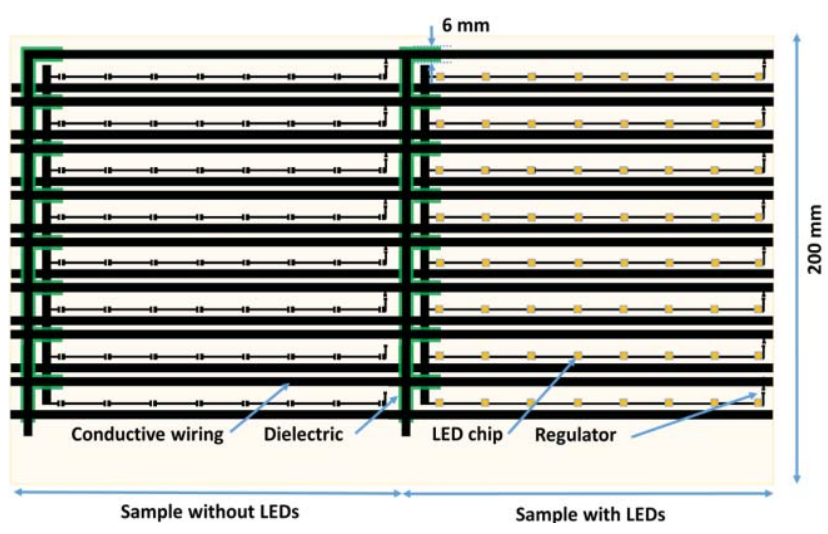

Fig. 1. Layout of the measured flexible electronics sample. Sample withoug LEDs is considered as 'Sample 1' and sample with LEDs as a 'Sample 2'.

\section{B. Synchronized Thermograhy Based Measurement System}

PI 640 IR camera (Optris $\mathrm{GmbH}$ ) was used to capture IR images. The camera is based on a two-dimensional uncooled focal plane array (UFPA) detector capable to measure IR emission of the heated object. The temperature measurement range covers $-20{ }^{\circ} \mathrm{C}$ to $900{ }^{\circ} \mathrm{C}$ in the spectral range of $7.5-13$ $\mu \mathrm{m}$. The camera resolution is $640 \times 480$ pixels with $32 \mathrm{~Hz}$ frame rate. The used camera lens had $33^{\circ}$ x $25^{\circ}$ field of view (FOV), enabling $0.075 \mathrm{~K}$ temperature resolution. The material and measurement conditions specific characteristic emissivity is adjustable between 0.100 and 1.100. The camera focusing is manually adjustable.

A direct current (DC) power source were used for electrical heating of the sample foils by using an ITT Instruments / Metrix AX 323 AC-to-DC power source. It has a total of three $0-30 \mathrm{~V}$ outputs with a maximum current output of $2.5 \mathrm{~A}$. The summary of used electrical powering parameters are summarized in Table I.

TABLE I. PARAMETERS OF ELECTRICAL HEATING

\begin{tabular}{|l|c|c|}
\hline Parameter & Sample 1 & Sample 2 \\
\hline Input voltage & $12 \mathrm{~V}$ & $24 \mathrm{~V}$ \\
\hline Input Current limit & $\begin{array}{c}0.5 \mathrm{~A} / 1 \mathrm{~A} \\
5 \mathrm{sec}(0.5 \mathrm{~A}) / \\
2 \mathrm{sec}(1.0 \mathrm{~A})\end{array}$ & \begin{tabular}{c}
$10 \mathrm{~mA}$ \\
\hline Heating time
\end{tabular} \\
\hline
\end{tabular}

The raw IR image data were exported from the PI Connect software for post-processing and analysis with Matlab.

\section{Data processing}

IR images were captured in 8-bit grayscale format, contrast-adjusted and finally pseudo-colored to make it easier for the human eye to analyze in case Sample 1.

In order to perform the automated analysis of LED chip heating during the powering, a specific image processing algorithm was implement in Matlab. At first, the input grayscale image was converted to a binary image. Next, the built-in function 'regionprops' was used to find out the centroid coordinates of the objects in the binary image. The resulting array includes the coordinates of the LEDs and the current regulators which exhibited the most intense heating behavior when powered. By employing the 'imdilate' function, a disk shaped areas closest the observed shape of
LED's was used to determine a heat emission of them. The radius was chosen appropriately depending on the distance from which the IR image was captured, so that the relevant sized area around each LED chip was included in the calculations. Finally, the numerical values of the emitted intensities were merged with the original grayscale image. The summary of the data processing flow is shown in Fig. 2.

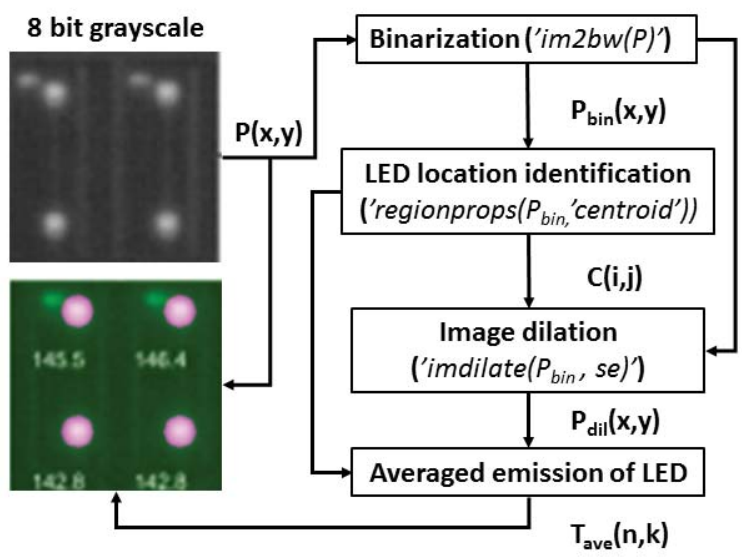

Fig. 2. Summary of the image processing algorithm used in analysis of Sample 2 .

\section{Topology measurement device}

The uniformity of the physical dimensions and the estimation of the thickness of conductive lines were measured with an optical profilometer (Bruker ContourGT$\mathrm{K} 0 \mathrm{X}$ ). Vertical scanning interferometry (VSI) mode was used in this study.

\section{MEASUREMENT RESULTS}

\section{A. Conductive wiring and dielectric uniformity analysis}

The ST measurement method was applied for analysing of heating behavior of the printed conductive structures. Measurements were performed for Sample 1 without any active electronics bonded on foil. In total, 28 conductive lines were heated and measured individually. The location of the heating electrodes on the sample and representative examples of obtained temperature profiles are shown in Fig. 3.
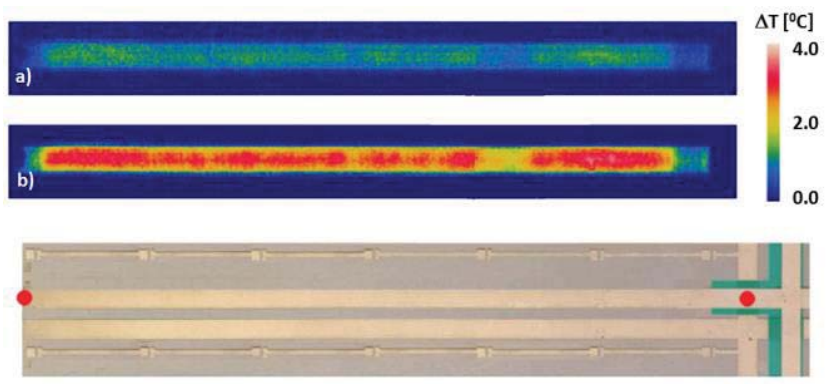

Location of heating electrodes

Fig 3. Temperature profiles of conductive lines. a) heating with 0.5 $\mathrm{A}$; b) heating with $1 \mathrm{~A}$ and the photography image of measured line with the location of heating probes. 
The ST measurements suggest that there are several areas present in the majority of the conductive lines, which have non-uniform conductivity properties. Some of these line sections were further studied with optical profilometer in order to find out if the line thickness varied at these locations. As seen in Fig. 3 a) and b) the electrical heating power and time have to be optimized depending on the used materials and structures to get reasonable contrast.

The ST measurement method was also utilized for analysing of heating behavior around the printed dielectric structures. The measurements were mostly focused on observing the heating effects at the regions around the interface of dielectric layers and conductive lines in Sample 1. The uniformity and structural integrity of these dielectric layers are important for the device in the electrical functionality point of view because the non-uniformities or

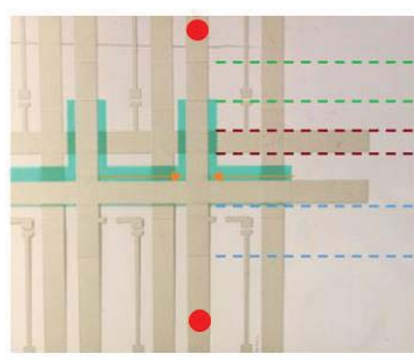

Power probe location

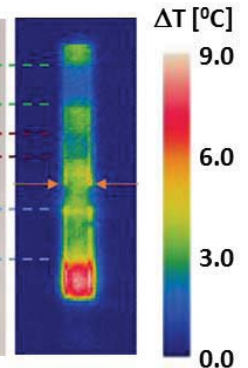

Fig 4. Temperature profiles of conductive lines on top of dieletric of the interconnection.

the defects in dielectric layers reduce the strength of them and could cause breakdowns when powered electrically. The dielectric layer is partly sandwiched between the conductive lines in the crossing section thus limiting the area to be characterized by ST methods.

Conductive lines beneath and above of the dielectric were electrically heated and temperature profiles were captured by ST. The obtained results are shown in Fig. 4 and 5. It is worth to mention that on the interconnection area, part of the conductive lines are having two overlapping conductive ink prints. In the Fig 4, the effect of double thickness is clearly seen in the ST image. The highest temperature is observed on the single print area (below blue dashed line) and the

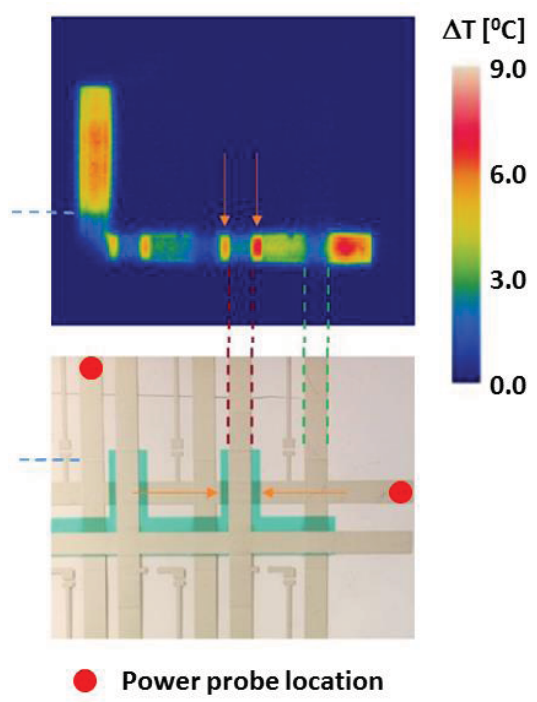

Fig. 5. Temperature profiles of conductive lines going underneath the dielectrics of the interconnection. double print right above it (between blue dashed lines). Once the thickness is decreased on a single print part, also the conductivity is decreased causing elevated temperature. In addition, a slight misalignment of the conductive ink prints can be seen in Fig. 5 (marked with orange arrows) causing increased temperature on the edge of the conductive area.

If the wire underneath the dielectric layer is heated (See Fig. 5.), the observed temperature signal is significantly increased on these areas. The reason for this observation is not clear.

\section{B. Conductive wiring structural analysis}

Physical dimensions of printed conductive structures are known to be proportional to their electrical resistivity [1] and thus the thickness variations of conductive lines were measured and compared to the results obtained from the ST measurements. The uniformity analysis of physical dimensions were done by measuring topology profile of the conductive lines from three separate sections of a conductive line by an optical profilometer. Two different sections were measured along the repetition of a printing pattern. As shown in Fig. 6. Section 1 is having the highest measured temperature increase while the Section 2 was the opposite with the lowest recorded temperature.

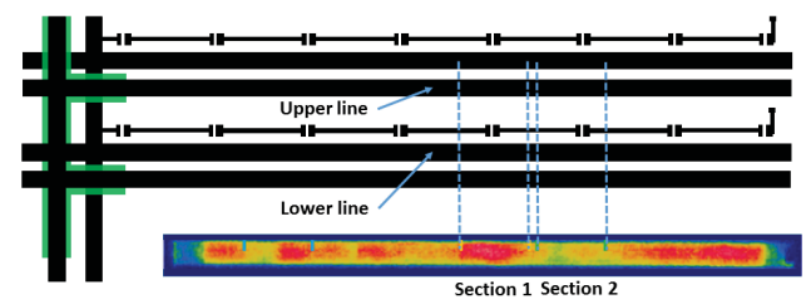

Fig. 6. Electrically heated temperature profile of the sections measured by profilometer.

The obtained thickness measurement values for those two sections are shown in Table II.

TABLE II. MEASURED THICKNESS OF CONDUCTIVE LINES

\begin{tabular}{|l|c|c|}
\hline Measured line & Section 1 $(\boldsymbol{\mu m})$ & Section 2 $(\boldsymbol{\mu m})$ \\
\hline Upper line, avg/med & $16.9 / 16.1$ & $27.8 / 22.8$ \\
\hline Lower line, avg/med & $20.6 / 20.6$ & $24,0 / 24.3$ \\
\hline
\end{tabular}

Measured thickness variations of the sections were relatively small but nevertheless consistent of the expected behavior. Once the thickness of silver conductor is increased, the resistivity is decreased causing lower current densities with same electrical heating power.

\section{Defect detection}

ST signal is not selective for different type of nonuniformities. That is why a visually observable defect was artificially created by removing some of the conductive wire by a knife thus reducing the cross-sectional area of the conductive line and increasing the current density on the remaining part of line. A clearly visible hot-spot with more than $10{ }^{\circ} \mathrm{C}$ temperature elevation can be seen above the crack (see Fig. 7). The result here discloses that any localized temperature increase of over $10{ }^{\circ} \mathrm{C}$ should be considered a major defect with the selected voltage and current settings. 


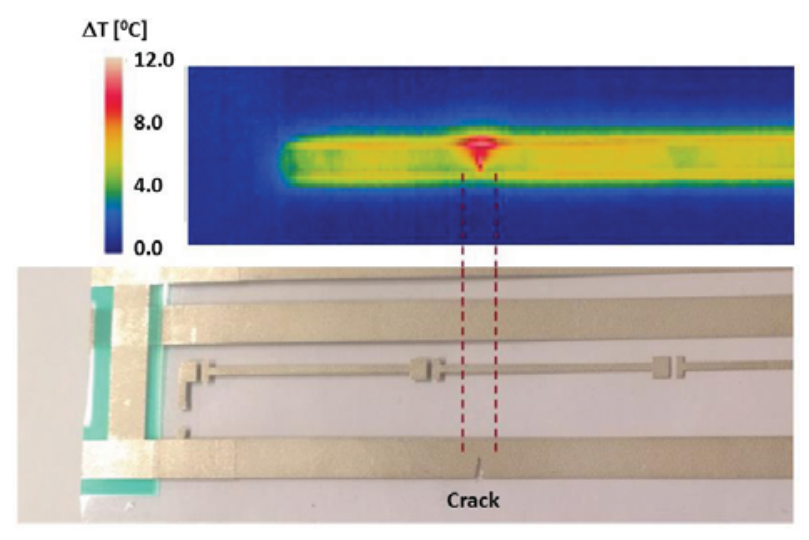

Fig. 7. Visually observable crack in a conductive line and its characterization by ST.

\section{LED chip characterization}

The sample with bonded LEDs and regulators (Sample 2) was examined by ST to analyze what kind of information could be seen while using the lightning element in normal operating mode. In contrast to Sample 1 measurement, additional challenges with the ST measurements were found due to different electrical and heating properties of the assembled components on the same sample. The temperature differences were relatively minor in case of Sample 1. However, in Sample 2 LEDs were heated much faster than the other structures, extending the dynamic range of measured temperature and thus affecting the contrast of obtained IR images. Due to that, performed ST measurements were practically limited to observing the heating behavior of the LED chips only. Even though the conductive lines used for bonding the LEDs were considerably narrower than the main conductive, the $25 \mathrm{~mA}$ LED driving current, allowed by the current regulator, is not high enough to induce significant heating contrast to IR emission of LED chips.

All the bonded 56 LEDs on the Sample 2 were characterized. An example of raw data images and processed LED heating characteristics covering the area of four LEDs close to the current regulators is shown in Fig. 8.
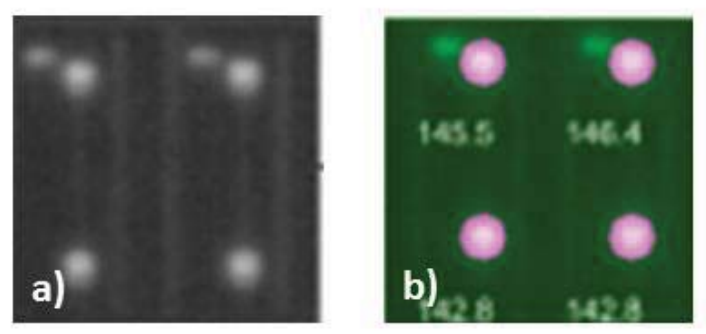

Fig. 8. IR images of four powered LEDs. a) Raw image data b) image processed data.

The statistical summary of the obtained results is presented in Table III. Based on the performed statistical analysis of heat emission, all the LEDs exhibited relatively similar heating behavior, indicating repeatable manufacturing process and good component quality. In addition, used current regulators seems to be heated in a similar manner as the LEDs.
TABLE III. MEASURED HEAT EMISSION OF LEDS

\begin{tabular}{|l|c|}
\hline Measured parameter & Sample 2 \\
\hline Emitted heat intensity, all LEDs (avg.) $[\Delta \mathrm{T}]$ & $2.6^{\circ} \mathrm{C}$ \\
\hline Min. LED heat emission (avg.) & $2.2^{\circ} \mathrm{C}$ \\
\hline Max. LED heat emission (avg.) & $3.0^{\circ} \mathrm{C}$ \\
\hline Min-Max emission difference, relative & $34.9 \%$ \\
\hline Peak temperature of LED & $28.9^{\circ} \mathrm{C}$ \\
\hline Peak temperature, current regulator & $28.8^{\circ} \mathrm{C}$ \\
\hline
\end{tabular}

More detailed analysis revealed that the maximum and the minimum heat emissions of the measured LEDs seems to be location dependent. The location of the most and least heated LEDs are depicted in Fig. 9.

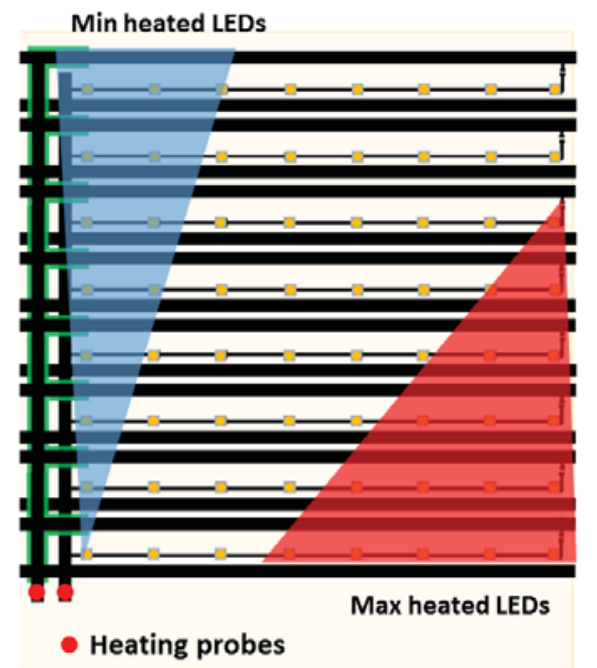

Fig. 9. Illustration of the most and least heated LEDs on the foil when powered.

\section{DISCUSSIONS}

The obtained results of the ST measurements are encouraging and consistent with expectations. Performed measurement results show that ST method enables the measurement of larger sample areas of thin film structures with bonded components, nicely complementing the existing characterization techniques suitable for this type of measurements.

The IR image pixel resolution and temperature resolution are heavily dependent on the specifications of the IR camera. The used PI 640 camera provided 640 x 480 pixels resolution with a temperature resolution of $75 \mathrm{mK}$ which are quite modest values for an IR camera. Despite of the limited performance of used camera, the obtained images has shown to be adequate for the described ST measurements and uniformity analysis purposes. In the studied case, the temperatures in ST measurements were relatively low, which means that IR detector with higher sensitivity at the lower end of the measurement temperature range should be preferred.

Identification of surface discontinuities in printed silver conductive lines was successful and the measurements provided spatial information on the current density distribution of the printed lines. In addition, the thickness variations of the conductive lines were clearly distinctive, especially in the proximity of the crossing sections, where 
the ends of the lines were overlapping due to the used screen printing technique. By employing proper heating parameters (time and input current), ST method could be utilized in the thickness estimation of printed conductive lines similar to the ones present in this study. However, thickness determination based on ST measurements requires pre-knowledge about the structure similar to previously published case with thin films on plastic [5]. In addition, the heat dissipation on the edges of the conductive lines has to be taken into account in thickness analysis.

One aim of this study was to analyze the bonding quality between the discrete components and the substrate based on ST imaging. Elevated temperature in the proximity of the bonding pads could be an indicator for poor bonding quality. Poor bonding quality will results in higher thermal and mechanical stress of the LED-substrate interface, predicting shorter lifespan for the LED-substrate bond. The LED device might still work as expected, but the connection between the chip and conductive lines on the substrate could suffer from delamination effects and loss of electrical contact causing eventually a failure of the device. Although some increased heating could be observed in the LED, the defective LED could not be identified from the thermal images as the heating behavior of all the LEDS were quite similar to each other. Increased heating indicates problem but the underlying causes and localization of the defective component only using the ST method would be with very difficult.

The used ST measurement system requires galvanic contact to the measured sample to be able to heat sample electrically. It is a well-known fact that a conductive structure can be heated by Eddy-current method in which varying electromagnetic field is used the generate current without contact to the sample [9]. That type of heating is expected to work for hybrid electronics samples as well. However, further investigation of this approach is needed.

One interesting approach to analyze ST data in more details could be the combination of ST measurements and the simulation of electrical and thermo-mechanical characteristics of hybrid electronics products such as the LED foil described in this study. For example, the effect of pn-junction heating during the powering and the effect of heat conduction on the foil could clarify many of the observed phenomena. That approach would enable the analysis the effect of environmental conditions on the lifetime of this type of products in various conditions to deepen the understanding of reliability of flexible, large area electronics [10-12].

\section{CONCLUSION}

The measurement configuration of ST is easy to setup and the measurement process is fast in comparison to many thin film measurements. In addition, the measureable area with an exposure is considerably larger than in other available characterization methods making ST promising especially for large area electronics applications. The ST measurement method has shown its potential as a characterization tool for conductive thin film structures but further analysis and system development are needed to exploit fully the potential of this technology.

\section{ACKNOWLEDGMENT}

We thank Technical Research Center of Finland Ltd (VTT) for providing the LED foil samples and technical advice for this research and Flexbright Oy for financial support to Antti Latomäki's Master's thesis work [13].

\section{REFERENCES}

[1] K. Keränen, P. Korhonen, J. Rekilä, O. Tapaninen, T. Happonen, P. Makkonen, and K. Rönkä, "Roll-to-rol printed and assembled large area LED lighting element," In J Manuf Technol, vol 81, pp. 529-536.

[2] O. Breitenstein, W. Warta, and M. Langenkamp, Lock-in Thermography: Basics and Use for Evaluating Electronic Devices and Materials, Springer, 2010.

[3] K. Leppänen, J. Saarela, R. Myllylä, and T. Fabritius, "Electrical heating synchronized with IR imaging to determine thin film defects," Opt. Express, Vol 21, pp. 32358-32370.

[4] K. Leppänen, J. Saarela, and T. Fabritius, "IR-imaging based system for detecting the defects of conductive materials," in Proc. 2014 SPIE Optical Engineering + Applications, Vol. 9205, 92050M.

[5] K. Leppänen, J. Saarela, and T. Fabritius, "Synchronized thermography for multilayer thin film characterization," in Proc. SPIE Thin Films for Solar and Energy Technology VI, Vol. 9177, 917705.

[6] C. Schuss, K. Leppänen, K. Remes, J. Saarela, T. Fabritius, B. Eichberger, and T. Rahkonen, "Detecting Defects in Photovoltaic Cells and Panels and Evaluating the Impact on Output Performances," IEEE Trans. Instrum. Meas., Vol. 65, pp. 1108-1119.

[7] J.-V. Voutilainen, T. Happonen, J. Häkkinen and T. Fabritius, "All silk-screen printed polymer-based remotely readable temperature sensor," IEEE Sensors Journal, Vo. 15, pp.723-733.

[8] K. Remes, K. Leppänen and T. Fabritius, "Thermography based online characterization of conductive thin films in large-area electronics fabrication," Optics Express, Vol. 26, pp. 1219-1229.

[9] J. García-Martín, J- Gómez-Gil and E. Vázquez-Sánchez, "Nondestructive Techniques Based on Eddy Current Testing," Sensor, Vol. 11 , pp. 2525-2565.

[10] T. Happonen, T. Ritvonen, P. Korhonen, J. Häkkinen and T. Fabritius, "Bending reliability of printed conductors deposited on plastic foil with various silver pastes," The international Journal of Advanced Manufacturing Technology, Vol. 82, pp. 1663-1673.

[11] T. Happonen, J. Häkkinen and T. Fabritius. "Cyclic bending reliability of silk screen printed silver traces on plastic and paper substrates," IEEE Transactions of Device and Materials Reliability, Vol. 15, pp. 394-401.

[12] T. Happonen, T. Ritvonen, P. Korhonen, J. Häkkinen and T. Fabritius, "Modeling the lifetime of printed silver conductors in cycling bending with the Coffin-Manson relation," IEEE Transactions on Device and Materials Reliability, Vol 16, pp. 25-29.

[13] A. Latomäki, "Synchtonized Thermography for Quality of Production Characterization of Flexible LED Foils," M.Sc thesis, Dept. Elect. Eng. , Univ. of Oulu, 2016. 